\title{
Fabrication of Aligned-Carbon-Nanotube-Composite Paper with High and Anisotropic Conductivity
}

\author{
Yuki Fujitsuka and Takahide Oya \\ Graduate School of Engineering, Yokohama National University, Tokiwadai 79-5, Hodogaya-ku, Yokohama 240-8501, Japan
}

Correspondence should be addressed to Takahide Oya, t-oya@ynu.ac.jp

Received 18 May 2012; Accepted 1 July 2012

Academic Editor: Magnus Willander

Copyright ( $) 2012$ Y. Fujitsuka and T. Oya. This is an open access article distributed under the Creative Commons Attribution License, which permits unrestricted use, distribution, and reproduction in any medium, provided the original work is properly cited.

\begin{abstract}
A functional carbon-nanotube (CNT)-composite paper is described in which the CNTs are aligned. This "aligned-CNT composite paper" is a flexible composite material that has CNT functionality (e.g., electrical conductivity) despite being a paper. An advanced fabrication method was developed to overcome the problem of previous CNT-composite papers, that is, reduced conductivity due to random CNT alignment. Aligning the CNTs by using an alternating current (AC) field was hypothesized to increase the electrical conductivity and give the paper an anisotropic characteristic. Experimental results showed that a nonionic surfactant was not suitable as a CNT dispersant for fabricating aligned-CNT composite paper and that catechin with its six-membered rings and hydrophilic groups was suitable. Observation by scanning electron microscopy of samples prepared using catechin showed that the CNTs were aligned in the direction of the AC field on the paper fibers. Measurement of the electric conductivity showed that the surface resistance was different between the direction of the aligned CNTs (high conductivity) and that of verticality (low). The conductivity of the aligned-CNT-composite paper samples was higher than that of nonaligned samples. This unique and functional paper, which has high and anisotropic conductivity, is applicable to a conductive material to control the direction of current.
\end{abstract}

\section{Introduction}

Nanotechnology, one of the most active research areas, has found application in many fields and has led to the development of various new carbon nanomaterials including fullerenes, graphenes, and carbon nanotubes (CNTs). CNTs, which were discovered in 1991 [1], are particularly attractive due to their various characteristics, including high electrical conductivity, high thermal conductivity, and two phases (semiconducting and metallic). Therefore, many researchers have studied everything from its growth technology to its application. Moreover, new materials have been developed that incorporate CNT characteristics. For example, the properties of materials like resin and aluminum have been improved by adding CNTs to them $[2,3]$. We previously developed a CNT-composite paper that is light, inexpensive, easily worked, and eco-friendly. Furthermore, it has the characteristics of CNTs despite being a paper and is thus potentially usable as an electrically conductive paper [4], an electromagnetic shielding sheet [5], a paper transistor [6], and a solar cell [7].
However, this CNT-composite paper was not well suited as an electrical conductive material due to its poor conductivity, which resulted from the random alignment of the CNTs on the fibers. The random alignment of the CNTs has a lot of contact points, which cause poor conductivity of the CNT-composite paper, among CNTs. To solve this problem, we have done this by aligning the CNTs, which reduced the number of contact points and created more straight conductive paths. Controlling the CNT alignment should improve the conductivity of the CNT-composite paper, resulting in an anisotropic characteristic.

\section{Fabrication of Aligned-CNT-Composite Paper}

We have previously reported a simple and easy method for fabricating CNT-composite paper [4]. We have now enhanced this method to enable control of the CNT alignment. Using this method, we can fabricate "aligned-CNTcomposite paper." In this study, we focus on controlling only 
CNT alignment on the surface of papers as a first step of achieving a fabrication of the new composite papers.

2.1. Trial Method. We decided to use an alternating current (AC) field to control the CNT alignment on the basis of a reported method showing that CNTs in dispersion can be aligned by using an AC field [8]. In that report, the CNTs were aligned in a manner similar to forming a bridge between electrodes. To fabricate aligned-CNT-composite paper, we considered the following four basic steps as a trial method.

(1) Preparing CNT dispersion. $5 \mathrm{mg}$ of multiwall CNTs (vapor grown carbon fiber; VGCF, Showa Denko Inc.) and $750 \mu \mathrm{L}$ of a nonionic surfactant (Triton X$100)$ as a CNT dispersant are used. They are added to $25 \mathrm{~mL}$ of pure water and irradiated the dispersion with ultrasonic waves for $30 \mathrm{~min}$.

(2) Preparing a square case $(2.5 \mathrm{~cm} \times 2.5 \mathrm{~cm})$, and place a sheet paper at the bottom of the case. Set the electrodes for applying the AC field facing each other on the sides of the paper. We here use a regular copier paper for simplicity.

(3) Dropping $1.5 \mathrm{~mL}$ of the CNT dispersion on the paper, and applying an AC field. Block pulse $E=40 \mathrm{~V} / \mathrm{cm}$ with $f=1 \mathrm{MHz}$ is applied.

(4) Continuing to apply the AC field until the CNT dispersion on the paper has dried.

We made a paper sample using this method and observed it using scanning electron microscopy (SEM). As shown by the SEM images in Figure 1, the CNTs were not aligned on the paper fibers despite the application of an AC field.

To determine the reason why the CNTs were not aligned, we investigated the penetrative properties of the CNT dispersion. As shown by the photograph in Figure 2, 10 seconds after being dropped, pure water had not penetrated the paper. In contrast, the CNT dispersion had penetrated the paper. This is attributed to the existence of the surfactant in the dispersion because a surfactant weakens surface tension.

We considered the movement of CNTs in the fabrication process. When the CNT dispersion was dropped on the paper, the CNTs were quickly adsorbed by the paper at random soon because, as Figure 2 shows, the CNT dispersion penetrated the paper. When the AC field was applied, the CNTs in the liquid layer may have been aligned. However, while drying, the aligned CNTs was affected to the randomly alignment CNTs adsorbed by the paper. As a result, the aligned CNTs were disturbed. We thus concluded that a nonionic surfactant should not be used for fabricating aligned-CNT-composite paper.

2.2. Revised Method. On the basis of this conclusion, we developed a method for controlling the CNT alignment that uses a nonsurfactant dispersant. We decided to use catechin from green tea (Figure 3 shows its structural formula) to disperse the CNTs [9] for two reasons.

(1) The six-membered ring of catechin fits that of CNT due to pi stacking interaction.

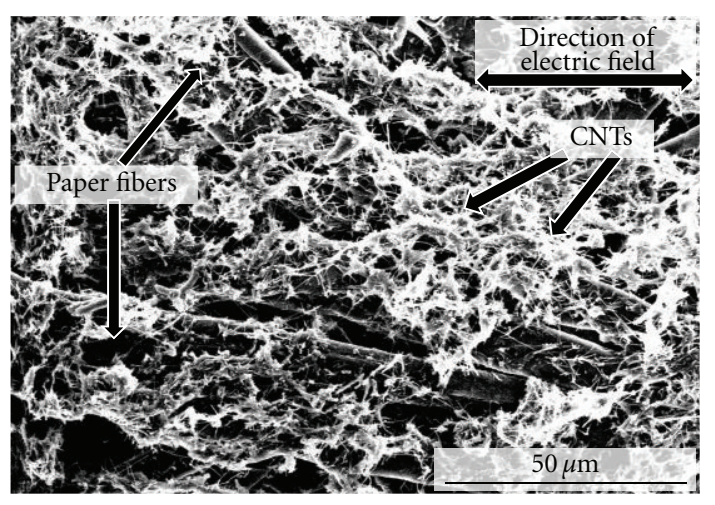

(a)

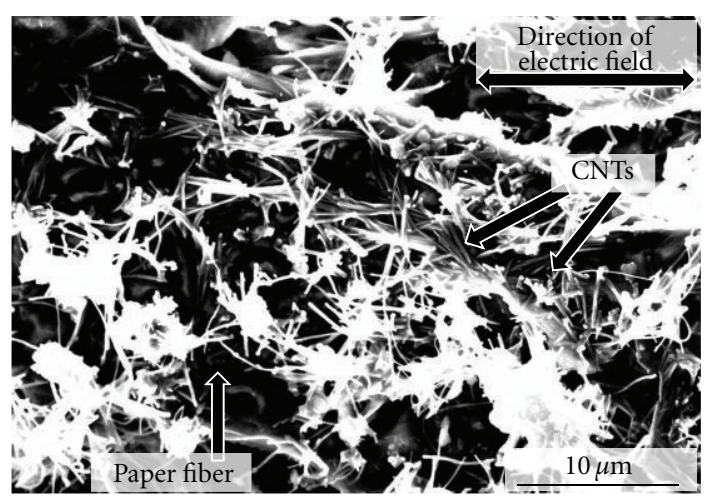

(b)

FIGURE 1: SEM image of CNT-composite paper ((a) low magnification, (b) high magnification). Dispersant was nonionic surfactant. CNTs were not aligned.

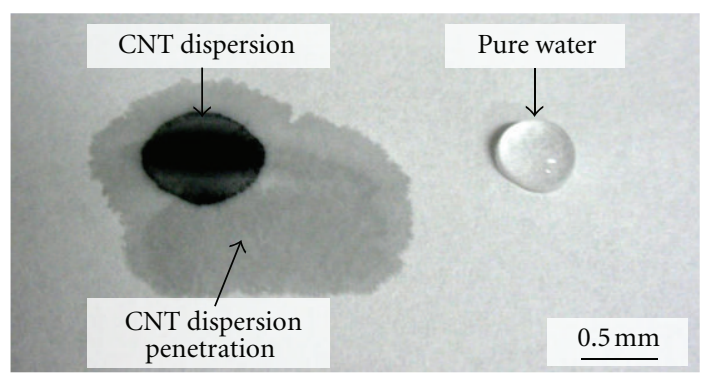

FIGURE 2: Appearance of CNT dispersion (left) and of pure water droplet (right) on paper 10 seconds after being dropped. CNT dispersion had penetrated the paper while pure water remained on the surface.

(2) Catechin can dissolve in water because it has a hydrophilic group.

We prepared a new CNT dispersion with catechin as the dispersant and dropped it on the paper. As shown in Figure 4, the dispersion had not penetrated the paper 10 seconds after being dropped.

We fabricated a sample by using the method described in Section 2.1 with catechin as the dispersant instead of a surfactant. We used $5 \mathrm{mg}$ of the VGCF and $5 \mathrm{mg}$ of the catechin. As shown by the SEM images in Figure 5, the CNTs on 


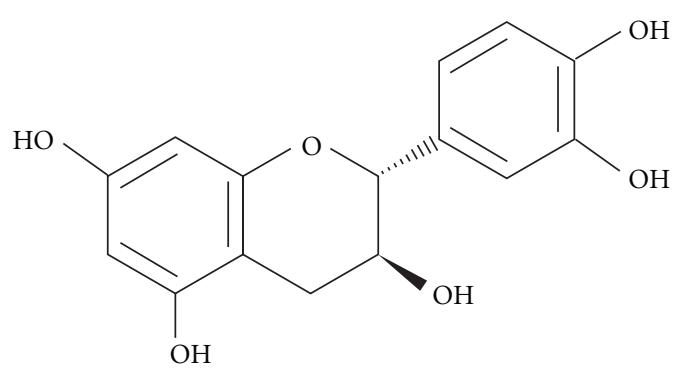

FIGURE 3: Structural formula of catechin with six-membered ring and hydrophilic group.

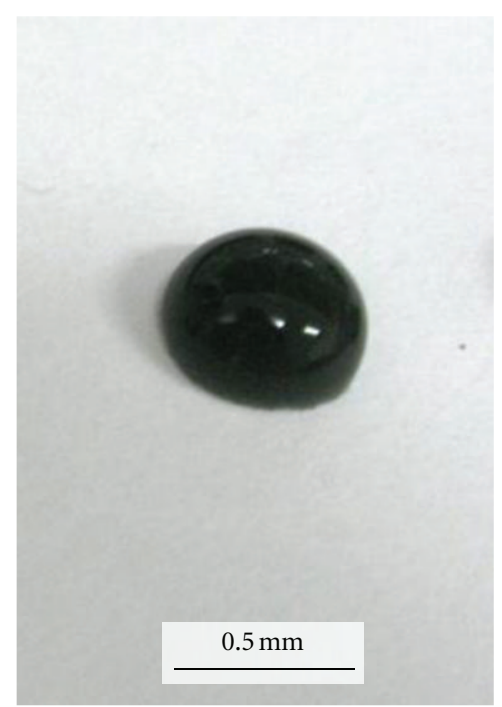

FIgure 4: Appearance of CNT dispersion with catechin on paper 10 seconds after being dropped. Dispersion did not penetrate the paper.

the paper fiber were aligned in the direction of the AC field. From these results, we concluded that catechin is suitable for fabricating aligned-CNT-composite paper. In this study, the fabricated samples are "aligned-CNT-covering paper" in the strict sense. However, our final goal is the development of "aligned-CNT-composite paper" that the aligned CNTs are placed in a paper. Therefore, we here assume the samples to be a one of the "aligned-CNT-composite paper."

\section{Conductive Property of Aligned-CNT-Composite Paper}

To clarify the role of the AC field in the alignment control, we measured the conductive property of the aligned-CNTcomposite paper. Specifically, we measured the surface resistance in two directions: the alignment direction and the vertical direction. For reference, we fabricated a nonalignment-controlled (AC field was not applied) CNTcomposite paper with the same CNT density by using the same method. For both samples, the CNT density on the paper was $0.0568 \mathrm{mg} / \mathrm{cm}^{2}$.

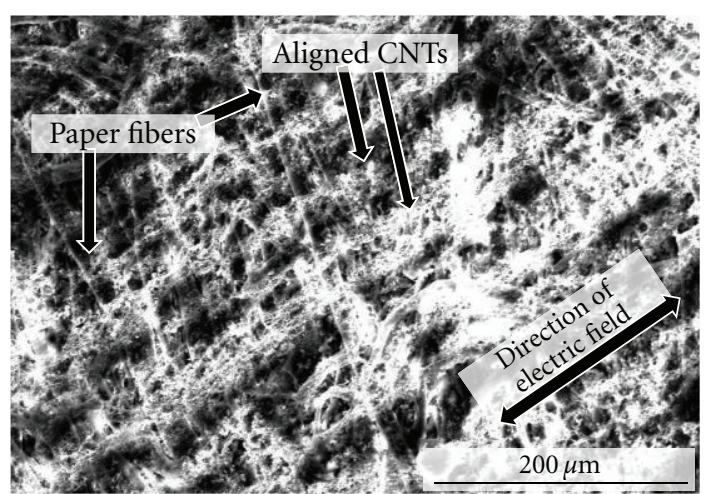

(a)

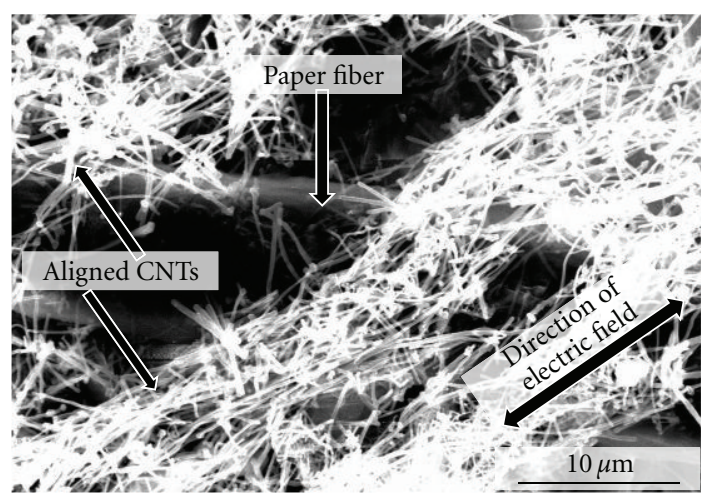

(b)

FIGURE 5: SEM image of CNT-composite paper ((a) low magnification and (b) high magnification). Dispersant was catechin. CNTs were aligned.

TABLE 1: Surface resistance of CNT-composite paper with CNT density of $0.0568 \mathrm{mg} / \mathrm{cm}^{2}$ (average for five measurements).

\begin{tabular}{lc}
\hline & Surface resistance $(\Omega /$ square $)$ \\
\hline Alignment direction & $2.86 \times 10^{3}$ \\
Vertical direction & $2.00 \times 10^{4}$ \\
Nonalignment control & $1.20 \times 10^{4}$ \\
\hline
\end{tabular}

As shown in Table 1, there was a difference in the resistance between the alignment direction and vertical direction. That is, the conductivity changed with the measurement direction. This means that aligned-CNT-composite paper has unique conductivity. Moreover, the conductivity in the alignment direction was higher than that for nonalignment control. These results show that the current flowed effectively in the CNT-composite paper, enabling the randomly aligned CNTs to be aligned. That is, we can align CNTs on paper and get high and anisotropic conductivity by using the proposed method.

Next, to investigate the relationship between conductivity and CNT density, we fabricated samples with five different CNT densities: 0.0284, 0.0426, 0.0568, 0.0852, and $0.1136 \mathrm{mg} / \mathrm{cm}^{2}$. We measured the surface resistance in two directions: alignment direction and vertical direction. Again we fabricated nonalignment controlled samples for 


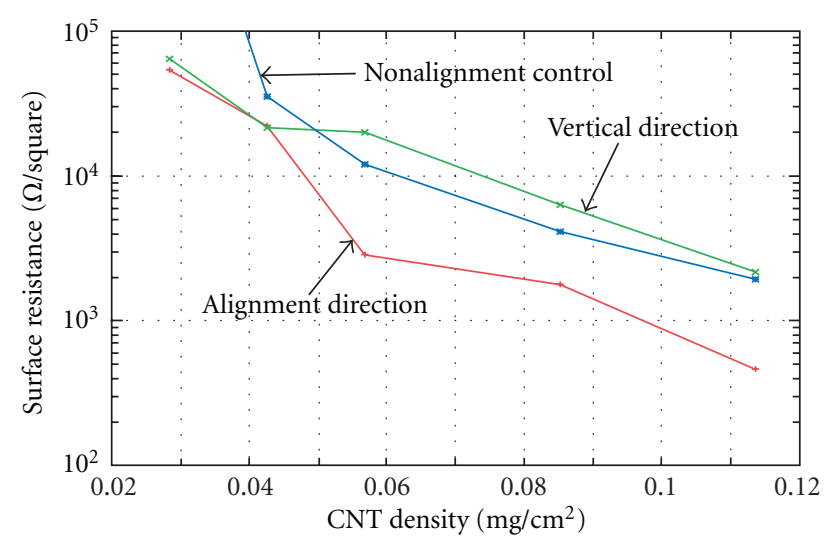

Figure 6: Surface resistance of CNT-composite paper (alignment direction, vertical direction, and nonalignment control).

reference. Figure 6 shows the surface resistance averaged over five measurements.

For the three samples with higher CNT density, conductivity increased in the following order:

vertical direction $<$ nonalignment control $<$ alignment direction.

For the two samples with lower CNT density, conductivity increased in the following order:

nonalignment control $<$ vertical direction $\approx$ alignment direction.

Conductivity was clearly dependent on CNT density. To clarify this dependency, we observed the sample with the highest CNT density $\left(0.1136 \mathrm{mg} / \mathrm{cm}^{2}\right)$ and the one with the lowest density $\left(0.0284 \mathrm{mg} / \mathrm{cm}^{2}\right)$ by SEM.

Figure 7(a) shows an SEM image of the results for the sample with the highest CNT density without alignment control. The paper fibers were indistinguishable due to the presence of many randomly aligned CNTs. Figure $7(\mathrm{~b})$ shows an SEM image with alignment control. The CNTs were aligned in the direction of the AC field. These results indicate that an AC field can be used to control the alignment of CNTs on paper.

Figure 8(a) shows an SEM image of the results for the sample with the lowest CNT density without alignment control. This sample did not exhibit conductivity because there were not enough CNTs on the paper fibers to construct a conductive path. Figure 8(b) shows an SEM image with alignment control. This sample did exhibit conductivity in both the alignment and vertical directions. The CNTs formed a striped pattern on the paper fibers and constructed a conductive path. We conclude that the AC field induced polarization in the CNTs, causing them to rotate and attract each other, resulting in the construction of a conductive path. These findings indicate that aligned-CNT-composite paper with fewer CNTs than previous CNT-composite papers can still be conductive.

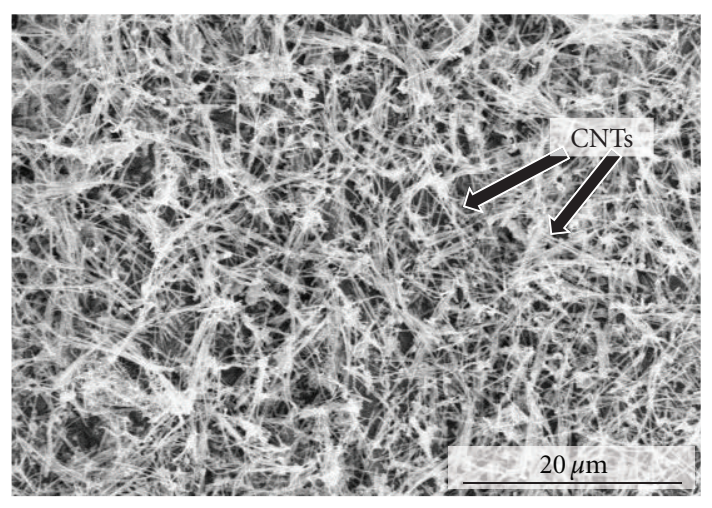

(a)

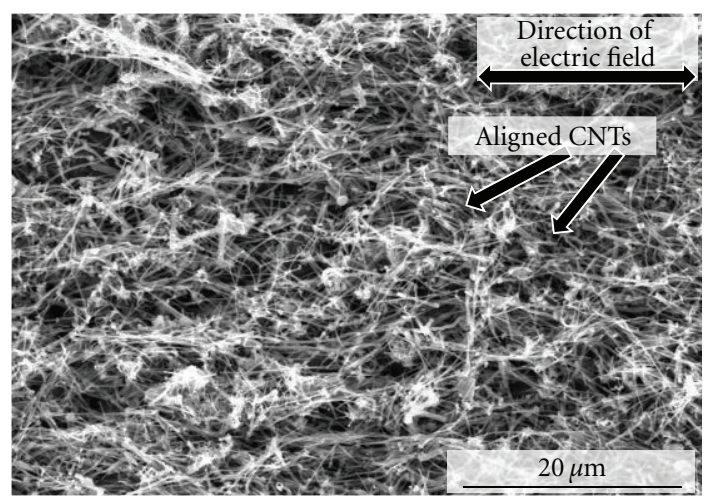

(b)

FIGURE 7: SEM image of CNT-composite-paper with CNT density of $0.1136 \mathrm{mg} / \mathrm{cm}^{2}$ ((a) nonalignment control and (b) alignment control). We could not confirm paper fibers because large number of CNTs hid them.

\section{Conclusion}

We have described a simple and easy method for fabricating aligned-CNT-composite paper by using an AC field. A CNT dispersion containing catechin is dropped on a paper, and an AC field is applied to the dispersion on the paper until it dries. The CNTs on the paper were aligned when catechin was used as the dispersant but not when a nonionic surfactant was used. Measurement of the conductive property of the aligned-CNT-composite paper showed that it had an anisotropic conductive property. This means that it can be used as a conductive material to control the direction of current. Moreover, its conductivity was higher than that of nonalignment-controlled-CNT-composite paper despite having the same number of CNTs. Therefore, aligned-CNTcomposite paper can be created using fewer CNTs. An investigation of the relationship between the conductive property and CNT density showed that the conductivity depends on the CNT density. This means that CNT-composite paper with fewer CNTs can be conductive if the alignment of the CNTs is controlled.

In this study, the resistance values of the fabricated samples were around a few $\mathrm{k} \Omega /$ square. These values seem to be high comparing with other CNT sheet values that have been reported. The reason we considered is existence of 


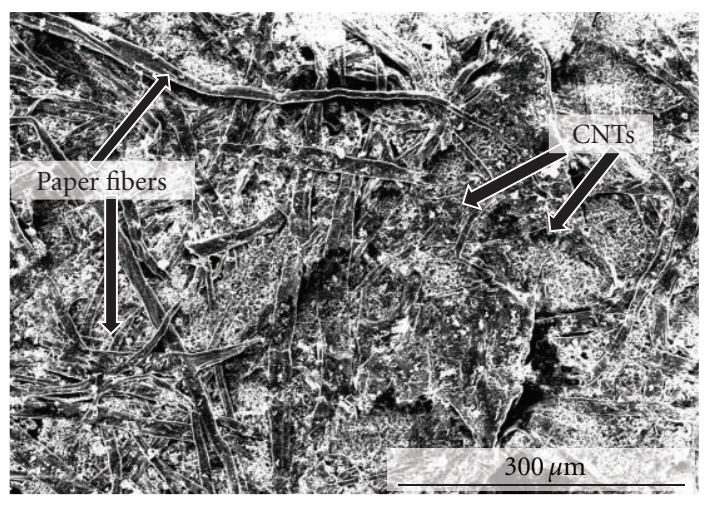

(a)

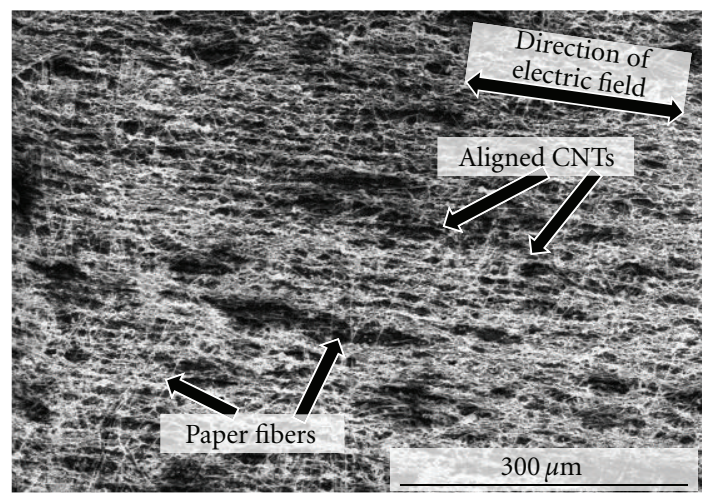

(b)

FIGURE 8: SEM image of CNT-composite-paper with CNT density of $0.0284 \mathrm{mg} / \mathrm{cm}^{2}$ ((a) nonalignment control and (b) alignment control).

catechin that may cover the VGCFs. Generally, the dispersant must be removed by evaporation via heating or by washing for fabrication of conductive CNT sheets to improve their conductivity. If we can remove the catechin dispersant, the resistance value should be reduced to around a few $\Omega$ /square. Because it has been reported that the bulk VGCF has about $10^{-3} \Omega \mathrm{cm}$ as an electrical resistivity [10]. As removal methods for catechin, a pyrolysis method [11, 12] and a washing method [13] have been reported, respectively. In the pyrolysis process, catechin is pyrolyzed from about $200^{\circ} \mathrm{C}$ by using steam $[11,12]$. However, such proposed methods can not be applied to our composite samples because the normal papers are used as substrates. The main component of the paper is cellulose. It is known that the cellulose is pyrolyzed from about $150 \sim 200^{\circ} \mathrm{C}$, so that the pyrolysis method must pyrolyze cellulose in addition to catechin. In contrast, the paper fibers may be deformed when they absorb water. Thus, the aligned CNTs may be disturbed by the deformed fibers, so that it is considered that the washing method is also not suitable for our composite. To improve the conductivity of our composite paper, a modified method should be studied as a next step.

Since CNTs have high thermal conductivity, a future work includes measuring the thermal conductivity of aligned-CNT-composite paper to determine its applicability to heat release materials. Because aligned-CNT-composite paper has an anisotropic conductive property, the direction of heat release should be controllable. Moreover, advanced fabricating method for the aligned-CNT-composite paper in the strict sense will be studied as a next interest.

\section{References}

[1] S. Iijima, "Helical microtubules of graphitic carbon," Nature, vol. 354 , no. 6348 , pp. 56-58, 1991.

[2] C. A. Martin, J. K. W. Sandler, A. H. Windle et al., "Electric field-induced aligned multi-wall carbon nanotube networks in epoxy composites," Polymer, vol. 46, no. 3, pp. 877-886, 2005.

[3] Y. F. Zhu, L. Shi, C. Zhang, X. Z. Yang, and J. Liang, "Preparation and properties of alumina composites modified by electric field-induced alignment of carbon nanotubes," Applied Physics A, vol. 89, no. 3, pp. 761-767, 2007.

[4] T. Oya and T. Ogino, "Production of electrically conductive paper by adding carbon nanotubes," Carbon, vol. 46, no. 1, pp. 169-171, 2008.

[5] M. Imai, K. Akiyama, T. Tanaka, and E. Sano, "Highly strong and conductive carbon nanotube/cellulose composite paper," Composites Science and Technology, vol. 70, no. 10, pp. 15641570, 2010.

[6] Y. Kawamura, S. Hayashi, Y. Shinde, and T. Oya, "Development of "Paper Transistor" using carbon-nanotube-composite papers," in Proceedings of the 4th International Conference "Smart Materials, Structures and Systems", DP-01, 2012.

[7] S. Sugiyama and T. Oya, in Proceedings of the 6th International Symposium on Surface Science and Nanotechnology (ISSS-6), 14PN-31, 2011.

[8] X. Liu, J. L. Spencer, A. B. Kaiser, and W. M. Arnold, "Electricfield oriented carbon nanotubes in different dielectric solvents," Current Applied Physics, vol. 4, no. 2-4, pp. 125-128, 2004.

[9] G. Nakamura, K. Narimatsu, Y. Niidome, and N. Nakashima, "Green tea solution individually solubilizes single-walled carbon nanotubes," Chemistry Letters, vol. 36, no. 9, pp. 11401141, 2007.

[10] M. Endo, Y. A. Kim, T. Hayashi et al., "Vapor-grown carbon fibers (VGCFs) - basic properties and their battery applications," Carbon, vol. 39, no. 9, pp. 1287-1297, 2001.

[11] S. Ohara, K. Hashida, R. Makino, and M. Kuroshima, "Japanese Unexamined Patent Application Publication (Japan Kokai Tokkyo Koho)," no. 2006-75070, March 2006.

[12] K. Nomizu, K. Hashida, R. Makino, and S. Ohara, "Antioxidants from steamed used tea leaves and their reaction behavior," Bioscience, Biotechnology and Biochemistry, vol. 72, no. 7, pp. 1682-1689, 2008.

[13] Y. Miyajima and H. Fuji, "Japanese Unexamined Patent Application Publication (Japan Kokai Tokkyo Koho)," no. 2009298625, December 2006. 

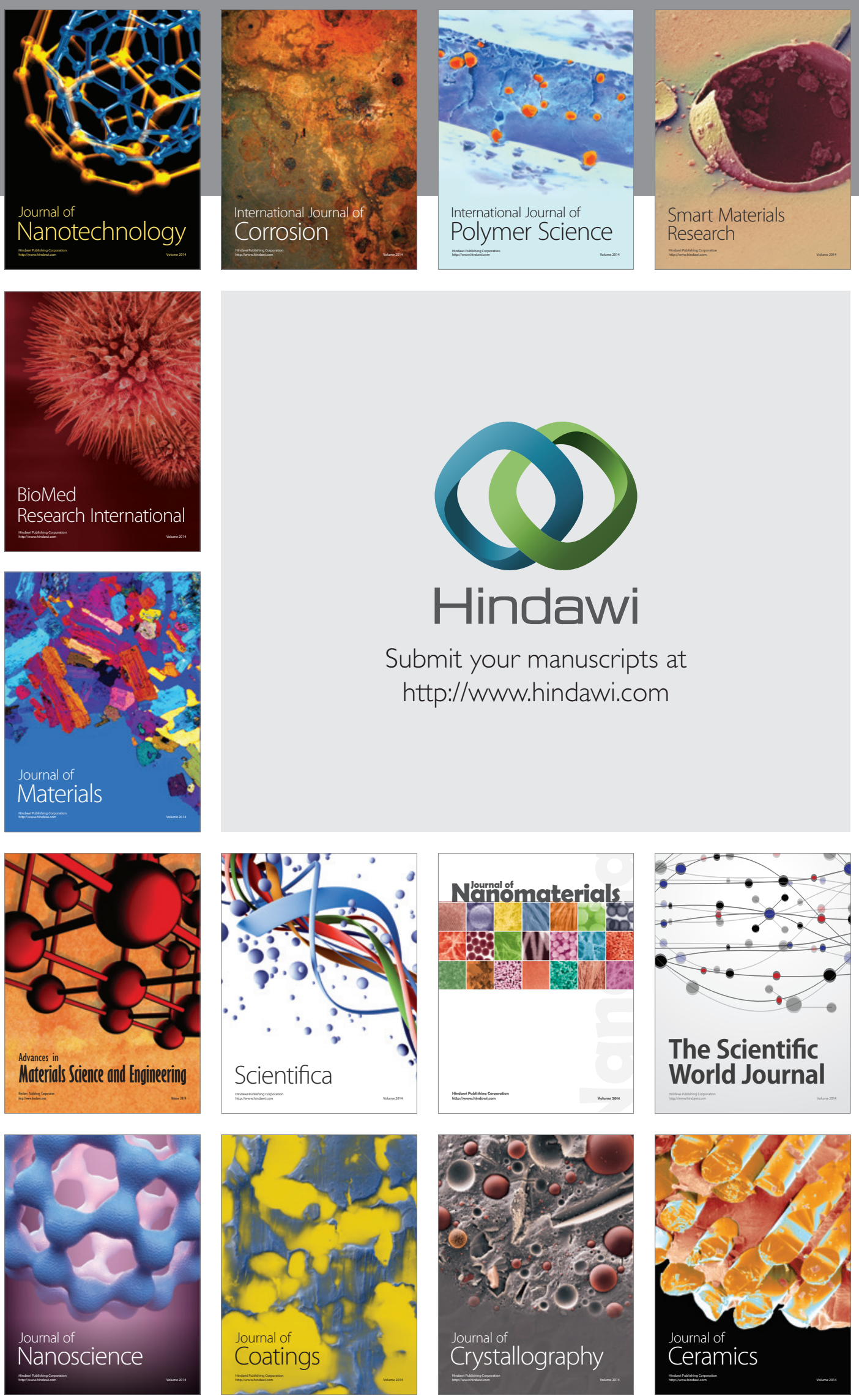

The Scientific World Journal

Submit your manuscripts at

http://www.hindawi.com

\section{World Journal}

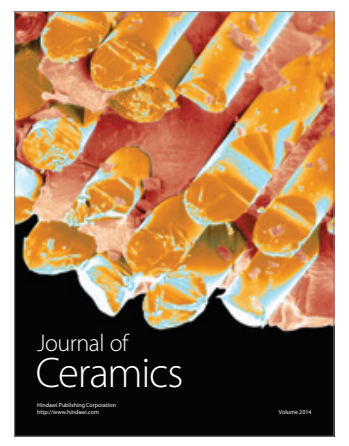

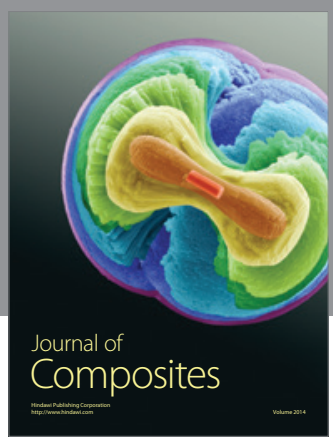
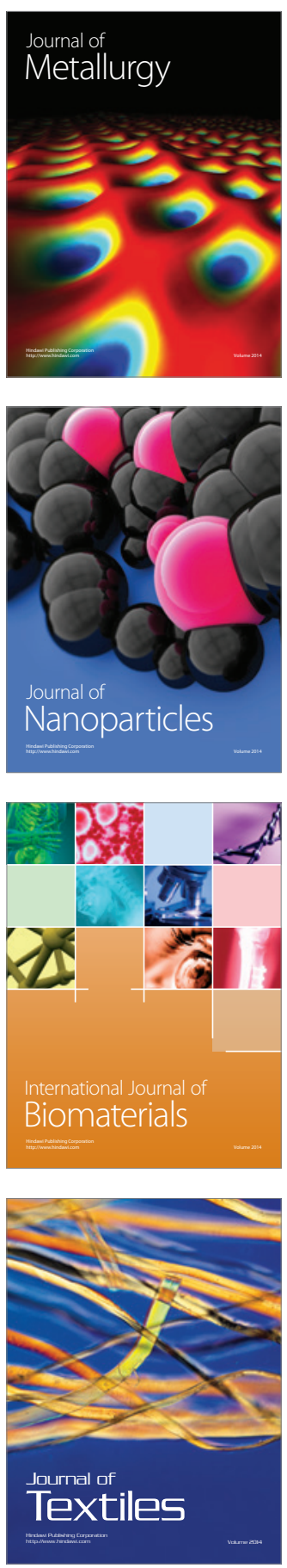